\title{
An accidental endoscopic finding - Trichuris trichiura. A case report and review of the literature
}

\author{
Shoket Chowdry ${ }^{1}$, Sandeep Dogra ${ }^{2}$, Bella Mahajan ${ }^{2}$ \\ ${ }^{1}$ Department of Gastroenterology, ${ }^{2}$ Department of Microbiology \\ Govt. Medical College \& Super Specialty Hospital, Jammu (J\&K), India
}

\begin{abstract}
Trichuris trichiura, commonly referred to as a whipworm, has a worldwide distribution, particularly among countries with warm and humid climates. This parasite is carried by nearly one quarter of the world population especially less-developed countries. Poor hygiene is associated with trichuriasis transmission, and children are especially vulnerable because of their high exposure to risk factors. This is especially true in developing countries, where poor sanitary conditions correlate with heavy disease burden and infections. Only patients with heavy parasite burden become symptomatic. The diagnosis is typically confirmed by detection of T. trichiura eggs on examination of a stool sample. This case report deals with a patient with trichuriasis who were diagnosed by detection of the parasite on colonoscopy. Thus colonoscopy might be a useful diagnostic tool, especially in symptomatic patients who are infected by only a few male worms with no eggs in the stool and thus are not diagnosed by conventional methods.
\end{abstract}

Keywords: Colonoscopy, trichuriasis, Trichuris trichiura

\section{INTRODUCTION}

Trichuris trichiura is a soil-transmitted helminth (STH) infection endemic in tropical and subtropical countries but few sporadic cases have occurred in nonendemic areas, mainly as a result of immigration. In India, the overall prevalence rates ranges from $13-66 \%$ with varying prevalence rates for individual parasites. ${ }^{[1]}$ Similar case reports have been seen in several developing countries like Malaysia, Nepal, Kuwait, and several parts of Kenya, Guyana and South Africa. $^{[2]}$

Corresponding Author: Dr. Shoket Chowdry

E-mail:shoketchowdry@gmail.com

Received: 29-08-2015

Accepted: 05-12-2015

How to cite this article: Chowdry S, Dogra S, Mahajan B. An accidental endoscopic finding-Trichuris trichiura. A case report and review of the literature. J Gastrointest Infect, 2015; 5: 54-56.
Most T. trichiura infections lack symptoms; only patients with heavy infections are symptomatic. The diagnosis is made by identifying T. trichiura eggs in stool specimens. However, the diagnosis of parasite infections by stool examination may be difficult in cases of infection with a few male parasites. Several reports have described the detection of $T$. trichiura during colonoscopy, both domestically and internationally. ${ }^{[3-6]}$ In this study a case report of a patient with massive trichuriasis accidentally diagnosed by detection of adult worms on colonoscopy is described along with review of such previously reported cases of trichuriasis in India.

\section{CASE REPORT}

A 35-year-old female reported to the out-patient department of Medicine, Government Medical College and Hospital, Jammu with a history suggestive of chronic large bowel non-bloody diarrhea, intermittent lower abdominal discomfort and tenesmus since 6 months. She had an unremarkable medical history. 


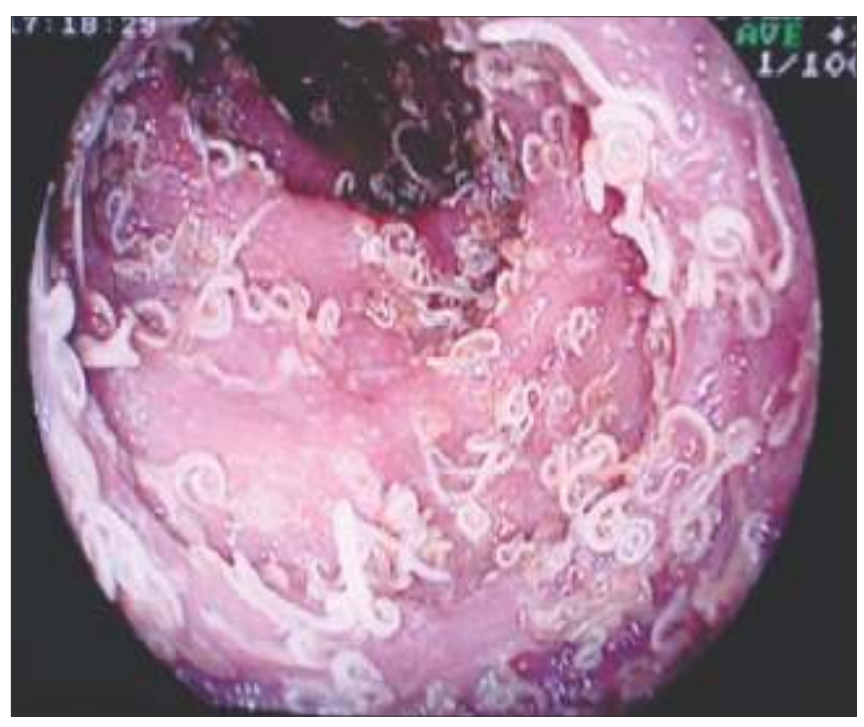

Fig.1 : Colonoscopy image capture showing live adult T. trichiura

Physical examination revealed pallor and per abdomen examination was also unremarkable. The laboratory investigations revealed: $\mathrm{Hb}, 8.7 \mathrm{~g} / \mathrm{dl}$ and $\mathrm{WBC}, 5,940 / \mathrm{cu}$ $\mathrm{mm}$. Platelet counts, ESR, electrolytes, liver and renal biochemical tests were within biological reference ranges. Mantoux test was non-reactive. Repeated stool examination for parasites or eggs was negative.

She was taken for colonoscopy which revealed numerous small, live, white colored worms in bunches smearing the colonic mucosa. One end of the collection was embedded in the hyperemic edematous mucosa and the other end was coiled and movable within the lumen. (Fig. 1) The worms were retrieved and sent to microbiologist for identification of species. Naked examination revealed worms to be about $3-4 \mathrm{~cm}$ in length, white in color and curved. Microscopic sectional evaluation of the retrieved parasite was consistent with the morphology of T. trichiura (a male worm including a testis). The patient was prescribed albendazole (400 mg orally for 3 days) and discharged. Repeated follow-up stool examinations over a period of six months revealed no parasites or eggs.

\section{DISCUSSION}

STH infections are one of the most important cause of morbidity especially in developing countries. The commonest parasitic infections reported globally are Ascaris (20\%), hookworm (18\%), T. trichiura (10\%) and Entamoeba histolytica (10\%). It is estimated that as much as $60 \%$ of the world's population is infected with intestinal parasites, which may play a role in morbidity due to intestinal infestations. Inadequate hygiene, poor health care systems/facilities, social indifference, social instability, civil war and natural disasters make the situations worse. ${ }^{[7]}$ STH are more important among children and in poor or malnourished populations. It has been shown to contribute to anemia, stunted growth, and underweight and poor school performance. $^{[2]}$

Trichuriasis is caused by ingesting embryonated eggs from the environment. Colonized eggs hatch and enter the crypts of the small intestine as larvae. After 1-3 months of maturation, the parasite migrates to the cecum. In the cecum, the parasite matures, mates, and lays eggs. Adult worms are 3-4 cm in length and have thin, tapered anterior regions, and are thus commonly referred to as whipworms. ${ }^{[-9]}$ The adult T. trichiura invade the mucosa and produce minor inflammatory changes at localized sites. In endemic areas, most people are colonized by small numbers of worms and have no symptoms. Some people harbor hundreds or even thousands of worms, and present with anemia, diarrhea, abdominal pain, weight loss, malnutrition, appendicitis, colonic obstruction, perforation, or intestinal bleeding. ${ }^{[8]}$ Treatment recommendations are as follows: albendazole (400 $\mathrm{g}$ once daily for 3 days) or mebendazole (100 mg twice daily for 3 days $).{ }^{[10]}$

In developed countries, detection of T. trichiura using colonoscopic examination is performed for immigrants from endemic areas for evaluation of their non-specific gastrointestinal symptoms, such as abdominal pain, diarrhea, and anemia. In recent years, direct inspection of whipworms at colonoscopy is increasing, presumably because of an increase in both the prevalence of STH and the use of colonoscopy. Further, colonoscopy screening has increased in recent years in both developed and developing countries for screening of colon cancers.

The patient was prescribed albendazole treatment after which her symptoms disappeared quickly, and repeated stool examinations carried over a period of six months failed to show any ova or parasite.

In the extensive review of literature conducted, it was found that the majority of publications were from South East Asian countries using modern available 
technology for screening and diagnoses of parasitic manifestations. Only one study of South India has reported similar scenario. To the best of our knowledge this is the first case report of T. trichiura diagnosed endoscopically from North India. The unusually heavy parasite burden makes it an interesting case report.

Although, stool examination is the method of choice for parasitic diagnoses, colonoscopy also might be a useful diagnostic tool, especially when infected with only a few male worms with no eggs in the stool examination and thus are not diagnosed by conventional methods. As colonoscopy becomes widespread, it would become remarkably important not only in the diagnosis and treatment of colorectal disease, but also in epidemiologic studies of parasite infections.

\section{CONFLICT OF INTEREST: None declared}

\section{ACKNOWLEDGEMENT}

The authors are grateful to the technical staff members of Department of Gastroenterology and Department of Microbiology for assistance in processing and documentation of parasite.

\section{REFERENCES}

1. Kang G, Mathew MS, Rajan DP, Daniel JD, Mathan MM, Mathan $\mathrm{VI}$, et al. Prevalence of intestinal parasites in Rural Southern India. Indian Tropical Medicine and International and Hygiene.1998; 80:706-18.

2. World Health Organization, Prevention and Control of intestinal parasitic Infections. Report of a WHO Expert Committee. World Health Organ Tec Rep Ser/ 1987; 749.

3. Chandra B, Long JD. Diagnosis of Trichuris trichiura (whipworm) by colonoscopic extraction. J Clin Gastroenterol. 1998;27:152-7.

4. Taguchi H, Yamamoto H, Miyata T, Hayashi Y, Sunada K, Sugano $\mathrm{K}$. In vivo diagnosis of whipworm (Trichuris trichiura) with highdefinition magnifying colonoscope (with video) Gastrointest Endosc. 2008;68:37.

5. Lin AT, Lin HH, Chen CL. Colonoscopic diagnosis of whipworm infection. J Gastroenterol Hepatol. 2005;20:965-7.

6. Chang CW, Chang WH, Shih SC, Wang TE, Lin SC, Bair MJ. Accidental diagnosis of Trichuris trichiura by colonoscopy. Gastrointest Endosc. 2008;68:154.

7. Elliott DE. Intestinal worms. In: Feldman M, Friedman LS, Brandt LJ, editors. Sleisenger \& Fordtran's Gastrointestinal and Liver Disease. 8th ed. Philadelphia, USA: Saunders; 2006. pp. 2441-2.

8. Lee SH, Chai JY, Hong ST. Synopsis of Medical Parasitology. 1st ed. Seoul, Korea: Korea Medical Book; 1996. pp. 62-6.

9. Keiser J, Utzinger J. Efficacy of current drugs against soiltransmitted helminth Infections: systemic review and metaanalysis. JAMA. 2008;299:1937-48.

10. Bundy \& deSilva DAP. Epidemiological aspects of Trichuris trichuriasis in Caribbean communities. Transaction of Royal Society of Tropical medicine Health, 1998; 3:70-75. 